\author{
Military Technical College \\ Kobry El-Kobbah, \\ Cairo, Egypt
}

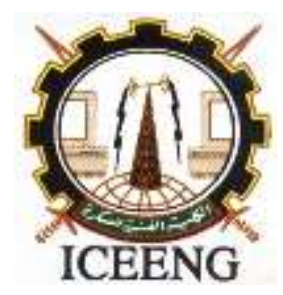

\author{
$11^{\text {th }}$ International Conference \\ on Electrical Engineering \\ ICEENG 2018
}

\title{
A Comparative Study of Supervised Classification Techniques for Multi-Spectral Images
}

\author{
Mahmoud abdallah Shwak* , Fawzy Eltohamy Hassan Amer ${ }^{*}$ \\ ABSTRACT \\ , Osama M. Mosa*, and Essam Hamza*.
}

Classification of satellite images is an important key for ground features extraction and thematic maps production. Satellite images with multi-spectral bands provide rich data which is useful for features extraction and description. Many supervised classification methods have been developed for classifying the multispectral images. Each method has its own advantages and disadvantages (limitations). In this paper the performance of four of the common used supervised classification techniques is compared. The techniques considered here are: Parallelepiped (PP), Minimum Distance (MD), Mahalanobies (MA), and Maximum Likelihood (ML). They are applied on a set of multispectral images acquired by Worldview-2 satellite. The classification results accuracy are analyzed and evaluated

The research work flow is processed by using ENVI. The developed maps are then visually compared with each other and accuracy assessments utilizing ground-truths. The assessment of classification results is represented in confusion matrix format and determination of Kappa coefficients. The preliminary results show that Maximum Likelihood (ML) gives accurate classification result for the area of study with overall accuracy $91.5741 \%$ and it is evaluated by Kappa coefficient which is 0.8846 :

\section{KEY WORDS}

supervised classification methods, Image classification assessment

\section{Introduction}

Satellite images play an important and vital role in providing geographical information for many branches and applications (Urban planning - Agriculture - Military applications - ...etc.) [1]. The advantages of satellite images are: they provide qualitative and quantitative information which reduces time for development and complexity of field work, they are collected at regular intervals and they are acquired

* Egyptian Armed Forces. 
in huge amount and still are growing with rapid rate [2, 3]. Satellite image classification is a process of grouping pixels into meaningful classes [4]. This process plays a vital role in interpretation and extraction of valuable information from volumes satellite images [7]. Urban area features can be extracted from satellite images by their classification using supervised or unsupervised classification methods [1]. The unsupervised classification also referred to as clustering attempts for an unclear grouping when no sample sets are available [5]. Supervised classification requires input from analyst and identifies different classes based on the sample training sets [6]. Supervised classification is more advantageous over unsupervised classification in most of the applications. In this work the performance of four of the common used supervised classification techniques is compared. The techniques considered here are: Parallelepiped (PP), Minimum Distance (MD), Mahalanobies (MA), and Maximum Likelihood (ML).

\section{Brief description of the selected four techniques}

\subsection{Concept of Parallelepiped classification technique}

Parallelepiped classification technique, sometimes also known as box decision rule, or level-slice procedures, is based on the ranges of values within the training data to define regions within a multidimensional data space as in Fig 1. The spectral values of unclassified pixels are projected into data space; those that fall within the regions defined by the training data are assigned to the appropriate categories [10]. The mean value (Mt) of all the pixels for a class $(\mathrm{C})$ for band $(\mathrm{M})$ is taken for all the $(\mathrm{N})$ classes of the training set and the variation (standard deviation) of the training data class (C) of band $\mathrm{M}$ of all the $\mathrm{N}$ classes be $\mathrm{St}$ as in equation (1). The mean and the standard deviation form the parallelepiped boxes as decision boundaries or intervals for assigning the pixels. A pixel will be assigned to a particular class if the digital number DN of the pixel lies inside the parallelepiped decision boundaries [11].

$\mathrm{Mt}-\mathrm{St} \leq \mathrm{DN}<\mathrm{Mt}+\mathrm{St}$

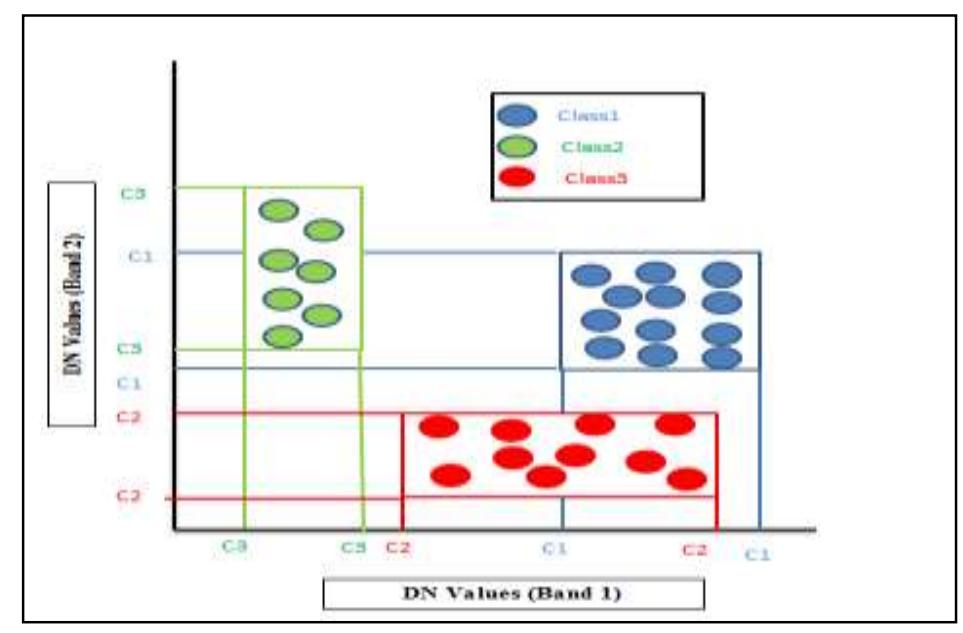

Fig.1: Concept of parallelepiped classification using two spectral bands 


\subsection{Concept of Minimum Distance Classification technique}

Each cluster can be represented by its centroid, often defined as its mean value. As unassigned pixels are considered for assignment to one of the several classes, the multidimensional distance [12] to each cluster centroid is calculated, and the pixel is then assigned to the closest cluster [10] as in Fig 2. This technique uses the distance measure, where the Euclidean distance is considered between the pixel values and the centroid value of the sample class. The pixel with the shortest distance with the class is assigned with that class [11]. The Euclidean distance is the usual manner in which distance is measured in the real world. On the other side, Manhattan distance tends to be more robust to noisy data.

$$
\text { Euclidean distance }=\sqrt{\Sigma_{i}\left(X_{i}-Y_{i}\right)^{2}}
$$

Where $\mathrm{x}$ and $\mathrm{y}$ are $\mathrm{m}$-dimensional vectors and denoted by $\mathrm{x}=\left(\mathrm{x}_{1}, \mathrm{x}_{2}, \mathrm{x}_{3} \ldots\right.$ am) and $\mathrm{y}$ $=\left(y_{1}, y_{2}, y_{3} \ldots y_{m}\right)$ represent them attribute values of two classes [13]. While Euclidean metric is useful in low dimensions, it doesn't work well in high dimensions and for categorical variables.

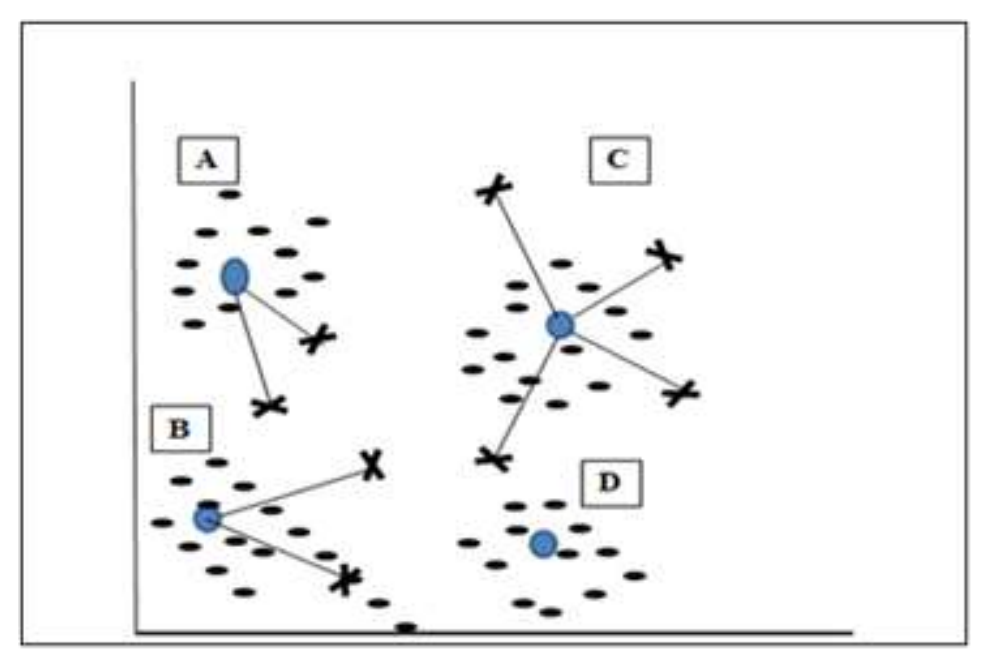

Fig 2: concept of minimum distance classifier

\subsection{Concept of Mahalanobies classification technique}

Mahalanobies distance is similar to minimum distance, except that the covariance matrix is used instead [14]. Unlike minimum distance, this method takes the variability of classes into account. It could be more useful than minimum distance in cases where statistical criteria must be taken into account, but the weighting factors that are available with the maximum likelihood option are not needed. However, this method tends to over classify signatures with relatively large values in the covariance matrix. Also, it is slower to compute than minimum distance; and it relies heavily on a normal distribution of the data in each input band. The Mahalanobies distance depends on the covariance matrix of the attribute and adequately accounts for the correlations as defined in equation (3). The covariance matrix is utilized to correct the effects of cross-covariance between two components of a random variable[15, 16] 


$$
\mathrm{D}=\left(\mathrm{X}-\mathrm{Mc}_{\mathrm{c}}\right)^{\top}\left(\mathrm{COV}_{\mathrm{c}}\right)^{-1}\left(\mathrm{X}-\mathrm{Mc}_{\mathrm{c}}\right)
$$

Where:

$\mathrm{D}=$ Mahalanobis Distance,

$\mathrm{C}=$ a particular class,

$\mathrm{X}=$ measurement vector of the candidate pixel

$\mathrm{Mc}=$ mean vector of the signature of class $\mathrm{c}$,

$\operatorname{Cov}_{\mathrm{c}}=$ covariance matrix of the pixels in the signature of class $\mathrm{c}$,

$\mathrm{Cov}_{\mathrm{c}}{ }^{-1}=$ inverse of Covc,

$\mathrm{T}=$ transposition function, [9].

\subsection{Concept of Maximum Likelihood classification technique}

Maximum Likelihood decision rule is based on the probability that a pixel belongs to a particular class. It can be described as a statistical approach to pattern recognition where the probability of a pixel belonging to each of a predefined set of classes is calculated; hence the pixel is assigned to the class with the highest probability [5]. The basic equation assumes that these probabilities are equal for all classes, and that the input bands have normal distributions as in equation (4): [17].

Where:

$$
\mathrm{D}=\ln (\mathrm{ac})-[0.5 \ln (|\operatorname{Covc}|)]-[0.5(\mathrm{X}-\mathrm{Mc}) \mathrm{T}(\operatorname{Cov}-1)(\mathrm{X}-\mathrm{Mc})]
$$

$\mathrm{D}=$ weighted distance (likelihood),

$\mathrm{C}=\mathrm{a}$ particular class,

$\mathrm{X}=$ measurement vector of the candidate pixel,

$M_{c}=$ mean vector of the sample of class $c$,

$\mathrm{a}_{\mathrm{c}}=$ percent probability that any candidate pixel is a member of class

$\mathrm{c}$,(Defaults to 1.0, or is entered from a priori knowledge),

$\mathrm{Cov}_{\mathrm{c}}=$ covariance matrix of the pixels in the sample of class $\mathrm{c}$,

$|\mathrm{Covc}|$ = determinant of Covariance (matrix algebra),

$\mathrm{Covc}^{-1}=$ inverse of Covariance (matrix algebra),

$L n=$ natural logarithm function $=$ transposition function (matrix algebra).

\section{Study Area}

The selected study area is a subset of Ismailia city - Egypt. Ismailia city was founded in 1863, during the construction of the Suez Canal, by Khedive Ismailia the Magnificent, after whom the city is named. It is the capital of the Ismailia Governorate, Egypt. The study area is at about $120 \mathrm{Km}$ to the north direction from Cairo the capital of Egypt. The study area is an urban area includes buildings, roads, vegetations, soil and water. It is approximates equal 91 hectares. 


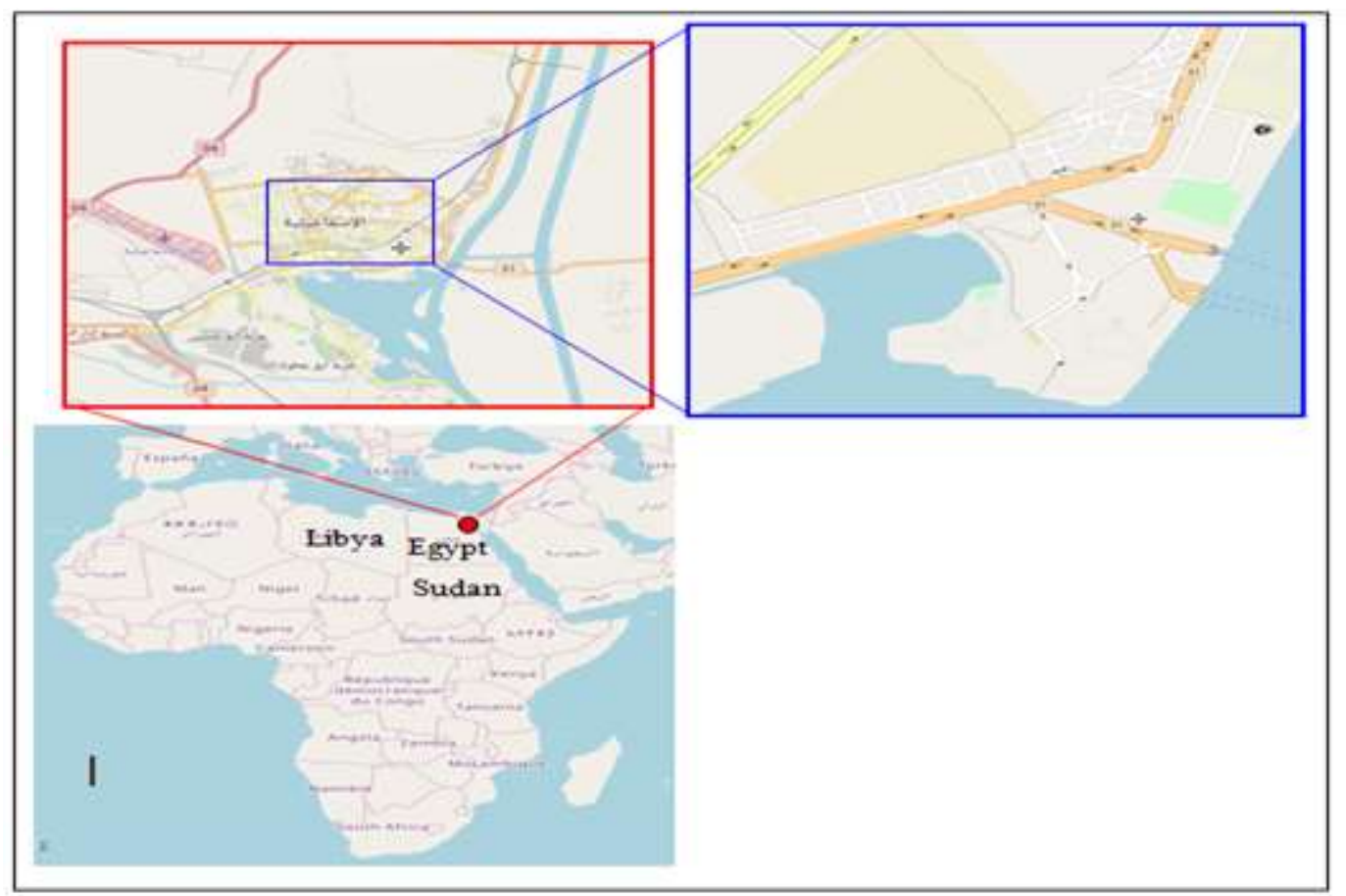

Fig. 3 Location of the selected area of Ismailia City in Egypt.

\section{Remote sensing data of study area}

In this work WV-2 satellite images are selected because WV-2 satellite provides four new spectral bands (Coastal blue, Yellow, Red edge and NIR-2), in Fig.4. These new bands give additional information for different features which can lead to better features extraction and hence better classification results accuracy [8]. The images data used are: PAN image of $0.5 \mathrm{~m}$ spatial resolution, Fig. (5), and 8-bands MS image of $2 \mathrm{~m}$ spatial resolution, Fig (6). Both images are captured on April 19th 2011. The projection of the images is UTM, zone 36. Spheroid and datum of the image is WGS 84. The upper left corner coordinates are (LAT30 ${ }^{\circ} 35^{\backslash} 44.3211^{\prime \prime} \mathrm{N}$, LON $32^{\circ}$ $17 \backslash 51.0860^{\prime \prime} \mathrm{E}$ ) and lower right corner coordinates of the image (LAT $30^{\circ} 35^{\prime}$ $16.0068^{\|} \mathrm{N}$, LON $32^{\circ} 18^{\backslash} 29.7392^{\|} \mathrm{E}$ ).

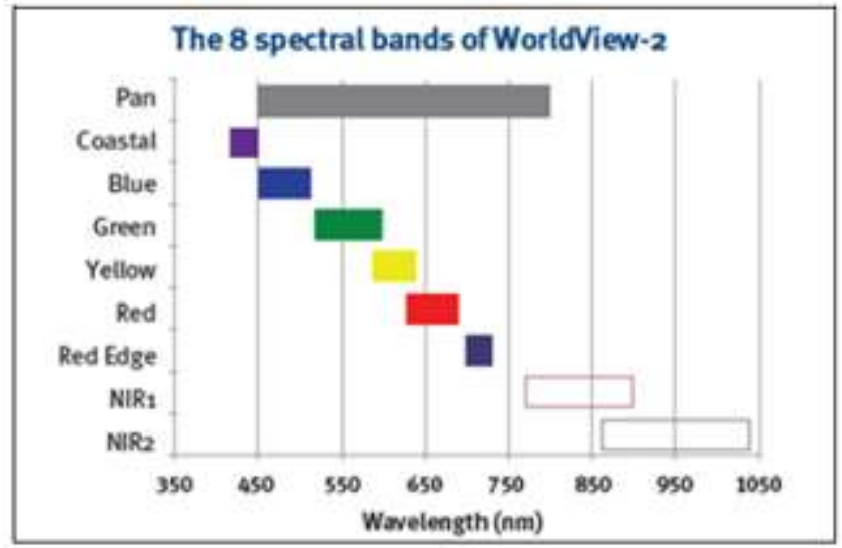

Fig. 4 the 8-Spectral bands of WV-2 


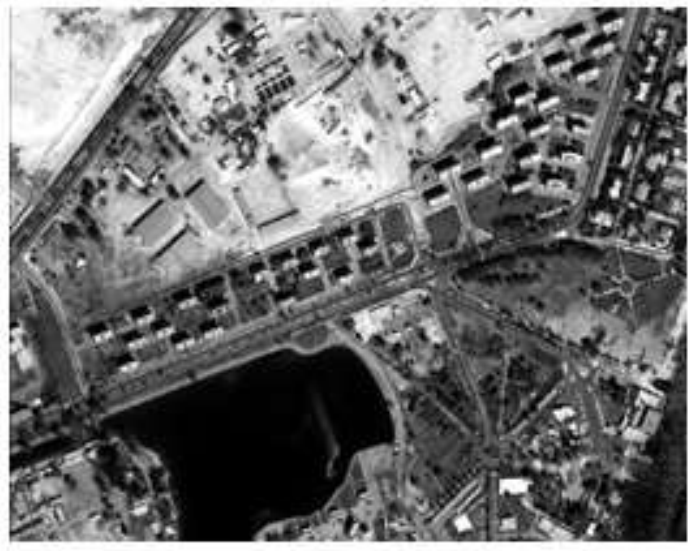

Fig. 5 PAN image

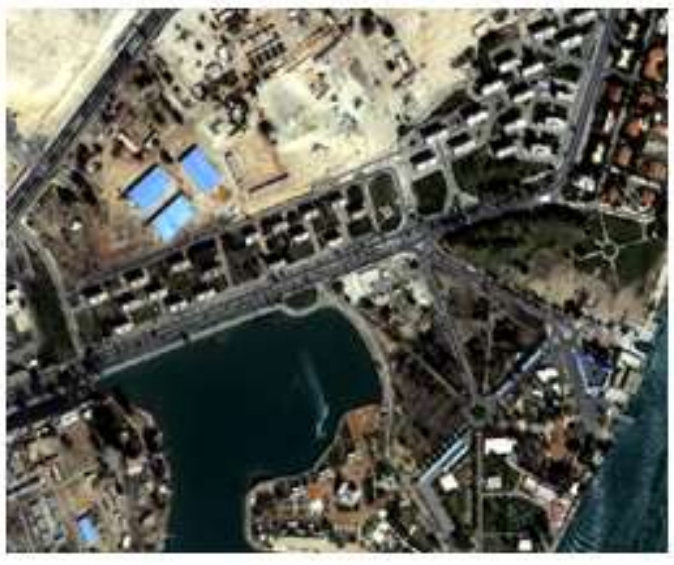

Fig. 6 MS Image

\section{Experimental work}

The experiment procedures are as given in Fig. (7):

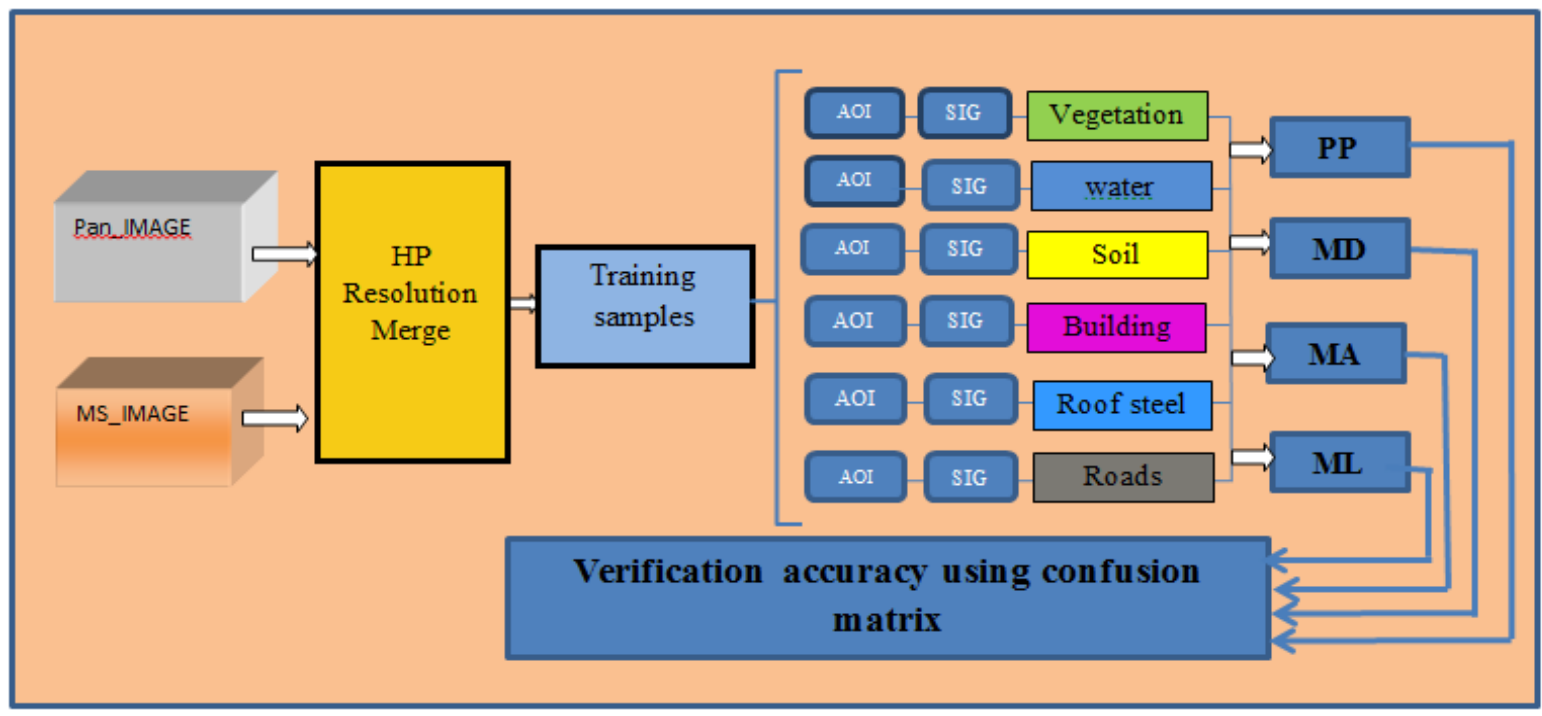

Fig. 7 : Experiment procedures

\subsection{Fusion process}

Original high spatial resolution panchromatic image $(0.5 \mathrm{~m})$ is fused with the low spatial resolution multispectral imagery $(8$ bands $-2 \mathrm{~m})$. The output fused image has 8-spectral bands with a spatial resolution of $(0.5 \mathrm{~m})$, Fig. (8). The "HP (High Pass) Resolution Merge" technique is used in fusion process. The process involves a convolution using a High Pass Filter (HPF) on the high spatial resolution data, then combining this with the lower spatial resolution multispectral data. The fusion process is performed using ERDAS_IMAGINE 2014. The result of this technique is a good details and a realistic representation of original multispectral scene colors [9] 


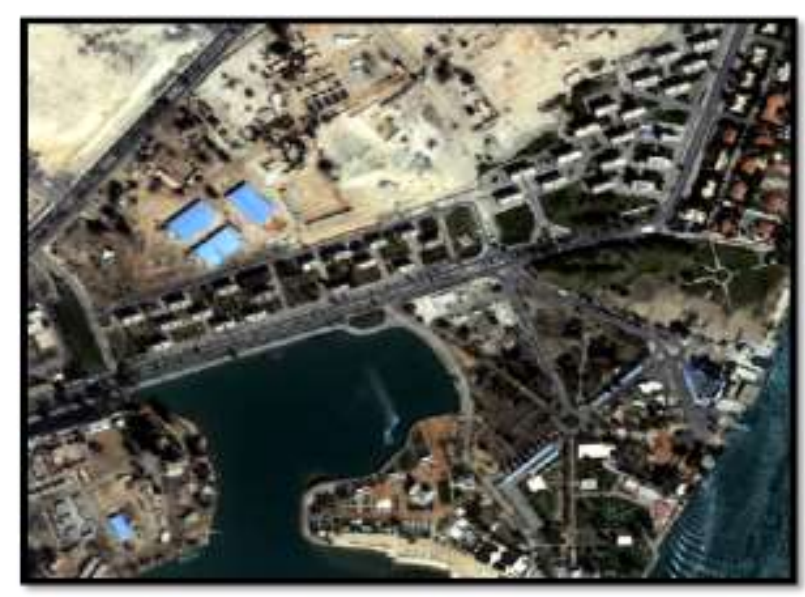

Fig. 8: Fused image

\subsection{Training samples selection}

A six training samples (vegetation, water, soil, building, steel roof, roads) are collected from fused image for the selected four supervised classifiers. In this work, 6 classes are present in the WV-2 imagery of the selected study area. The samples are extracted from the data set by using ENVI tool. The training samples are given in Table.1.

Table 1 Data of training samples for classes

\begin{tabular}{l|l|c|l|l}
\hline $\begin{array}{c}\text { Land cover } \\
\text { class }\end{array}$ & $\begin{array}{c}\text { Number } \\
\text { of pixels }\end{array}$ & $\begin{array}{c}\text { Number } \\
\text { of polygons }\end{array}$ & Area $\left[\mathrm{m}^{2}\right]$ & color \\
\hline \hline Water & 424.718 & 2 & $106,179.500$ & blue \\
\hline \hline vegetation & 254.378 & 69 & $63,594.500$ & green \\
\hline \hline Building & 47.414 & 69 & $11,853.500$ & magenta \\
\hline \hline Soil & 509.014 & 29 & $127,253.500$ & yellow \\
\hline \hline Steel roof & 21.731 & 3 & $5,432.750$ & cyan \\
\hline \hline Roads & 87.734 & 24 & $21,933.500$ & gray \\
\hline \hline
\end{tabular}

It is important to mention that the performance of many classifiers depends on the selection of sample size, number of features and the complexity of classifier. If number of sample size increases, then number of features also increases which in result the high complexity [18] [19]. The selection of sample size depends on the following factors

- Number of training sites for samples collection

- Method of sampling

- Data source for labelling training sites 
- Timing of data collection

\subsection{Applying the selected four classification techniques}

The four selected classifiers: Parallelepiped, minimum distance, mahalanobies distance and maximum likelihood are applied respectively on the WV-2 fused imagery data using ERDAS-Imagin 2014 SW package. The classifications results are given in figures (9 to 12)

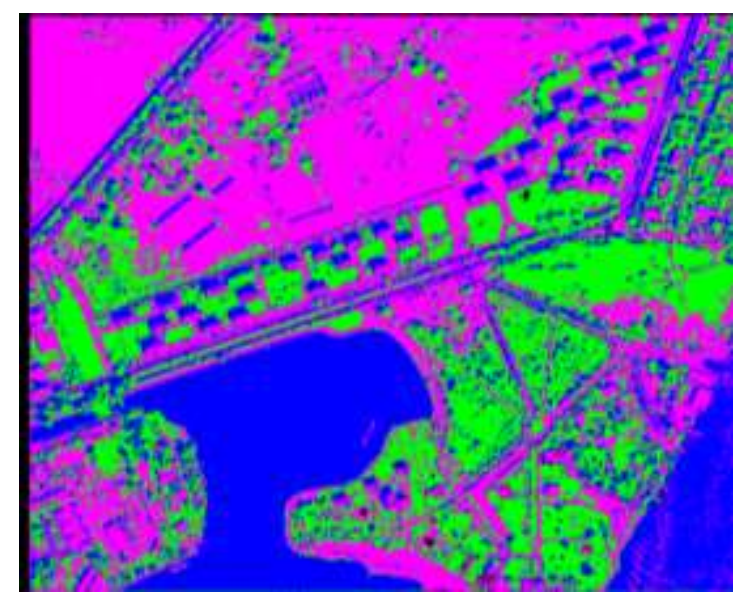

Fig.9: Parallelepiped classification result

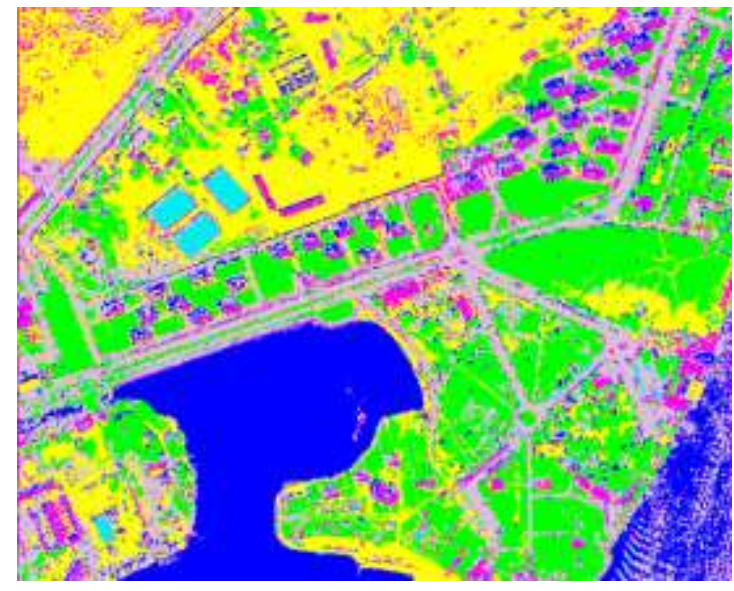

Fig.11:mahalanobies distance classification result

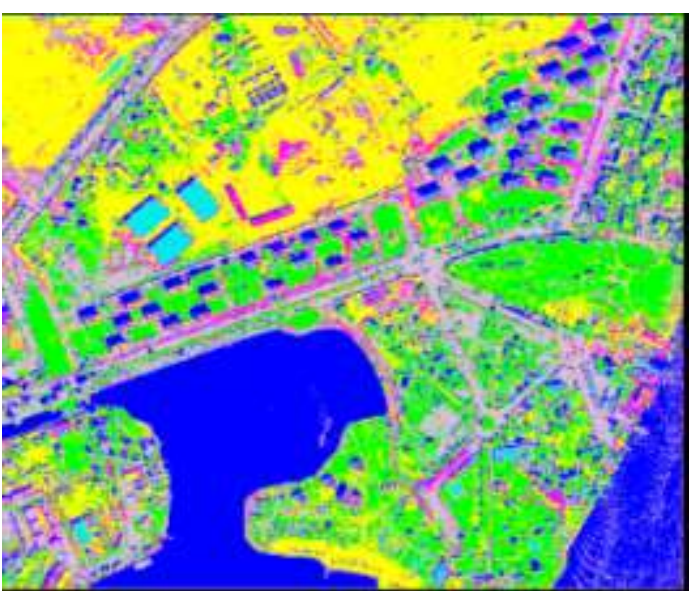

Fig.10: Minimum distance classification result

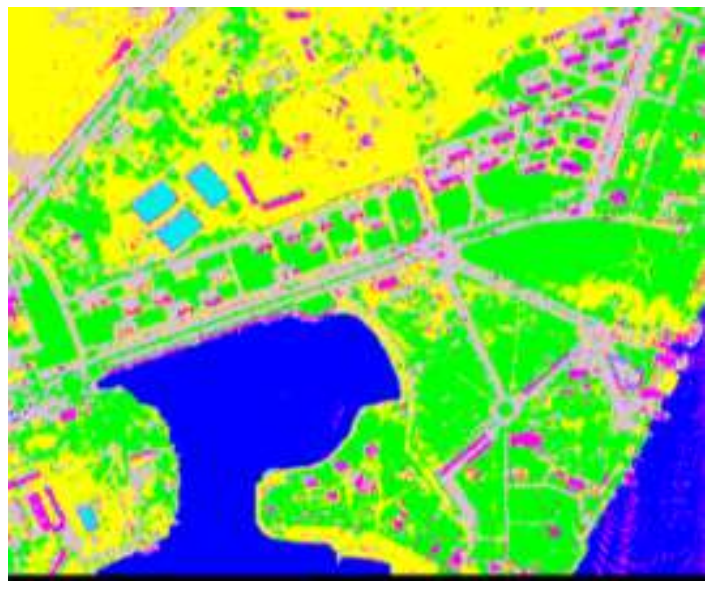

Fig.12. Maximum likelihood classification results

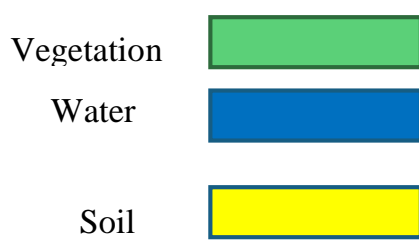

\section{Building}

Steel roof

Roads
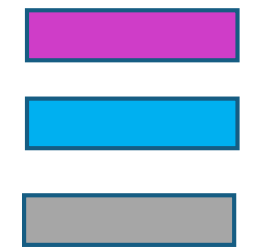


\subsection{Classification results accuracy assessment}

Accuracy assessment of the classification results is calculated by means of confusion matrix (sometimes called error matrix). It computes the relationship between results of a classification and the corresponding reference data (ground truth) .We obtain from accuracy assessment report that contains producer and user accuracies, an overall accuracy, kappa coefficient . where Overall Accuracy $(O A)$ is calculated by summing the number of pixels classified correctly and dividing by the total number of pixels, Producer's accuracy (PA) is calculated by dividing total number of correct pixels in a class by the total number of pixels of that class as considered from the reference data(ground truth) to measure correctly classification of reference data, User's accuracy (UA) is calculated by dividing the total number of correct pixels in a class by the total number of pixels that were classified in that class corresponds to measure probability of pixel classified on the thematic image actually represents that class on the ground and kappa coefficient $(K)$ is another method for measuring accuracy of classification which is calculated by subtracting Chance agreement incorporates off-diagonal from Observed accuracy determined by diagonal in error matrix and divided by subtracting Chance agreement from one[12]. Calculation of accuracy assessment of each classified image is applied by Envi software and defines the area of interest (AOI) of each class as reference data. For WV-2 dataset, the confusion matrix and kappa coefficient for the selected techniques are given in tables (2 to 5 )

Table (2) Accuracy assessment with ground truth (Percent) using PPC

Overall Accuracy $=(683248 / 1344989)=50.7995 \%$

Kappa Coefficient $=0.4078$

\begin{tabular}{|l||c||c|c||c||c|c|c||c|c||}
\hline class & water & Vegetation & Building & soil & roads & $\begin{array}{l}\text { Steel } \\
\text { roof }\end{array}$ & total & PA & UA \\
\hline unclassified & 0.11 & 0.32 & 0.92 & 0.06 & 0.12 & 0 & 0.16 & & \\
\hline water & 97.39 & 10.26 & 4.61 & 1.49 & 41.46 & 0 & 36.1 & 97.3 & 85.1 \\
\hline \hline vegetation & 0 & 88.53 & 0.79 & 9.77 & 11.94 & 0 & 21.2 & 88.5 & 78.8 \\
\hline Building & 2.5 & 0.90 & 93.68 & 88.6 & 46.48 & 100 & 42.4 & 93.6 & 7.78 \\
\hline Soil & 0 & 0 & 0 & 0 & 0 & 0 & 0 & 0 & 0 \\
\hline Roads & 0 & 0 & 0 & 0 & 0 & 0 & 0 & 0 & 8.7 \\
\hline Steel roof & 0 & 0 & 0 & 0 & 0 & 0 & 0 & 0 & 0 \\
\hline
\end{tabular}


Table (3) Accuracy assessment with ground truth (Percent) using MDC

Overall Accuracy $=(1090926 / 1344989)=81.1104 \%$

Kappa Coefficient $=0.7449$

\begin{tabular}{|l||l||l||l|l|l|l|l||l||l|}
\hline class & water & Vegetation & Building & soil & roads & $\begin{array}{l}\text { Steel } \\
\text { roof }\end{array}$ & Total & PA & UA \\
\hline \hline unclassified & 0 & 0 & 0 & 0 & 0 & 0 & 0 & & \\
\hline \hline water & 95.25 & 6.33 & 3.36 & 0.59 & 9.57 & 0 & 32.24 & 95.2 & 93.29 \\
\hline vegetation & 0 & 75.32 & 0.42 & 6.32 & 3.15 & 0 & 16.86 & 75.3 & 84.50 \\
\hline Building & 0.5 & 0.04 & 42.23 & 7.11 & 6.03 & 1.64 & 4.76 & 42.2 & 31.25 \\
\hline Soil & 0 & 5.03 & 17.46 & 75.70 & 3.41 & 0.06 & 30.44 & 75.7 & 94.12 \\
\hline Roads & 4.04 & 13.27 & 14.27 & 9.43 & 77.62 & 0 & 12.92 & 77.6 & 39.19 \\
\hline Steel roof & 0.22 & 0 & 22.26 & 0.85 & 0.21 & 98.2 & 2.78 & 98.2 & 57.17 \\
\hline
\end{tabular}

Table (4).Accuracy assessment with ground truth (Percent) using MAC

Overall Accuracy $=(1176196 / 1344989)=87.4502 \%$

Kappa Coefficient $=0.8304$

\begin{tabular}{|c|c|c|c|c|c|c|c|c|c|}
\hline class & water & Vegetation & Building & soil & roads & $\begin{array}{l}\text { Steel } \\
\text { roof }\end{array}$ & Total & $\overline{\text { PA }}$ & UA \\
\hline unclassified & 0 & 0 & 0 & 0 & 0 & 0 & 0 & & \\
\hline water & 91.45 & 0.65 & 4.55 & 0.94 & 2.41 & 0 & 29.67 & 91.4 & 97.32 \\
\hline vegetation & $\overline{0}$ & $\overline{97.42}$ & 0.08 & 3.40 & 1.25 & $\overline{0}$ & 19.79 & 97.4 & 93.08 \\
\hline$\overline{\text { Building }}$ & $\bar{~} 1.11$ & $\overline{0.05}$ & $\overline{70.07}$ & 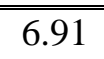 & 2.79 & 0.04 & 25.63 & $\overline{770.0}$ & 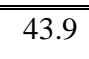 \\
\hline 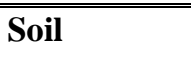 & "0.06 & 0.67 & $\overline{18.44}$ & 80.13 & 5.42 & $\overline{0}$ & 31.48 & 80.1 & 96.35 \\
\hline Roads & 7.1 & 1.23 & $\overline{5.45}$ & 8.58 & 87.97 & 0.09 & 11.65 & 87.9 & 499.24 \\
\hline Steel roof & .28 & $\overline{00}$ & $\overline{1.40}$ & 0.03 & 0.16 & 999.8 & 1.78 & $\overline{999.8}$ & 90.89 \\
\hline
\end{tabular}


Table (5).Accuracy assessment with ground truth (Percent) using MLC

Overall Accuracy $=(1231661 / 1344989)=91.5741 \%$

Kappa Coefficient $=0.8846$

\begin{tabular}{|l||c||c||c|c|c|c|c||c||c||}
\hline class & water & Vegetation & Building & soil & roads & $\begin{array}{l}\text { Steel } \\
\text { roof }\end{array}$ & Total & PA & UA \\
\hline \hline unclassified & 0 & 0 & 0 & 0 & 0 & 0 & 0 & & \\
\hline water & 95.76 & 0 & 2.17 & 0.02 & 0.03 & 0 & 30.33 & 95.7 & 99.72 \\
\hline vegetation & 0.04 & 98.57 & 0.60 & 3.8 & 2.24 & 0 & 20.26 & 98.5 & 92.02 \\
\hline Building & 3.97 & 0.04 & 78.80 & 3.04 & 2.2 & 0.24 & 5.34 & 78.8 & 52.06 \\
\hline Soil & 0 & 1.13 & 14.22 & 85.99 & 7.21 & 0 & 33.73 & 85.9 & 96.49 \\
\hline Roads & 0.21 & 0.27 & 3.86 & 7.15 & 88.3 & 0 & 8.72 & 88.3 & 66.07 \\
\hline Steel roof & 0.02 & 0 & 0.35 & 0 & 0.02 & 99.7 & 1.63 & 99.7 & 98.68 \\
\hline
\end{tabular}

\section{Analysis of accuracy assessment results}

Maximum Likelihood techniques achieve the highest values of accuracy and the better classification with overall accuracy of $91.5741 \%$ and kappa coefficient 0.8846 . The Mahalanobies classification also gives suitable values, although these were lower than those from Maximum Likelihood techniques. As for, The Parallelepiped techniques results were significantly lower than the other techniques. Fig. (13) Of OA and Fig. (14) Of K Illustrate results for classification techniques.

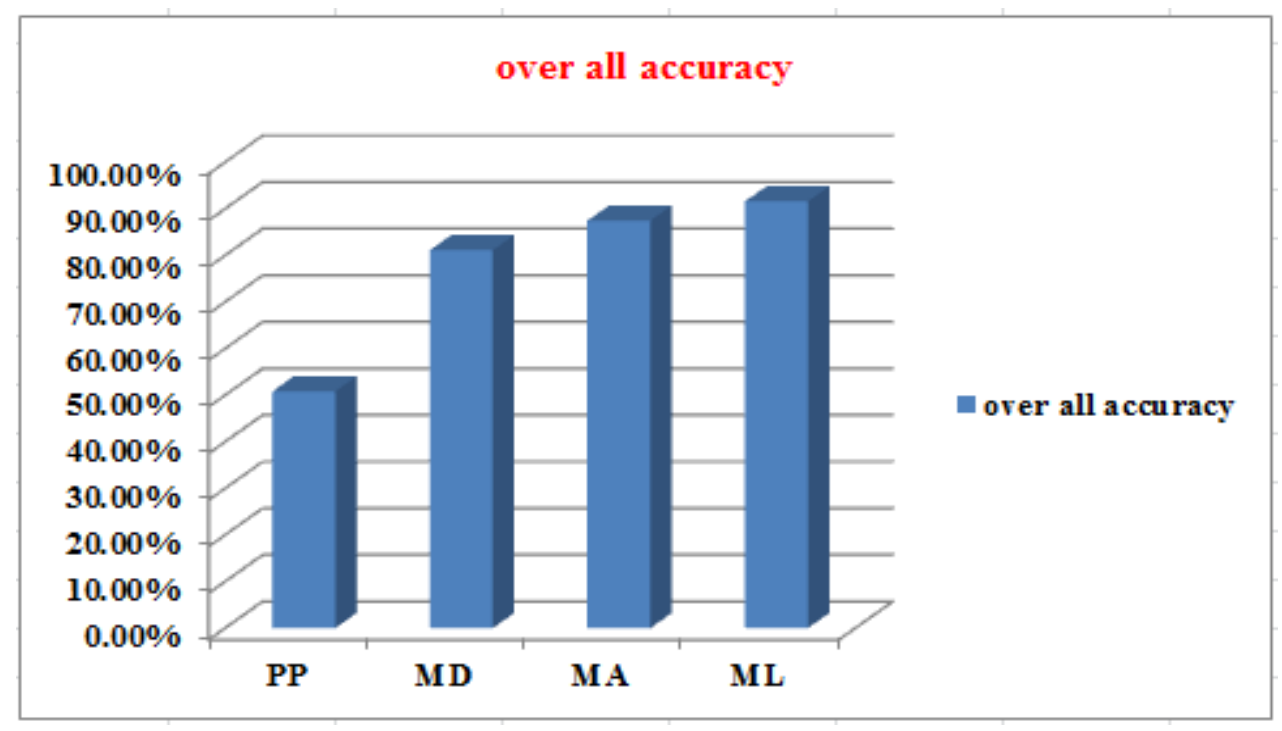

Fig. 13 over all accuracy of classification results of the selected techniques 


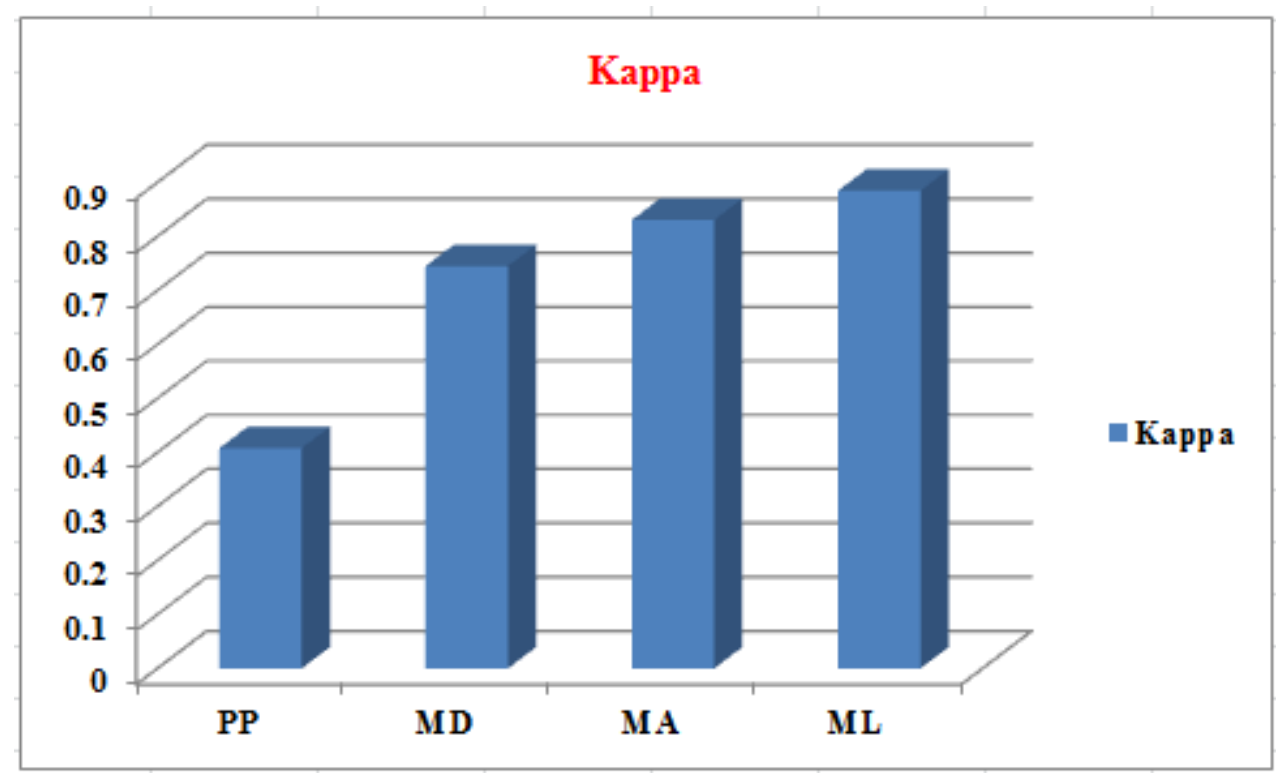

Fig. 14 Kappa for classification techniques

\section{Conclusions}

The different image Classification algorithms Parallelepiped, minimum distance, mahalanobies distance and maximum likelihood have been implemented and tested with ground truth. According to the results it can be stated that among all the supervised algorithms discussed generally maximum likelihood gives good results as compared to other algorithms in terms of accuracy but it has high time complexity. It cannot be said which classification algorithm best comparable to others as it depends on various factors as well as applications. There is tradeoff between time complexity and accuracy; for example, maximum likelihood provides very good result but takes much more time as compared to others. But if one wants average accuracy and faster results, then mahalanobies distance can be a better choice Moreover, the accuracy of algorithms also depends on the quality and robustness of training dataset. The selection of the dataset used and different environmental settings also adds up to the performance of the classification accuracy. Hence, the choice of a particular algorithm is made which fulfils the required parameters.

\section{Reference}

[[1] M. Shahbaz, A. Guergachi, A. Noreen, and M. Shaheen, "Classification by object recognition in satellite images by using data mining," in Proceedings of the World Congress on Engineering, 2012, pp. 4-6.

[2] C. Vaiphasa, S. Piamduaytham, T. Vaiphasa, and A. K. Skidmore, "A Normalized Difference Vegetation index (NDVI) Time-series of idle agriculture lands: A preliminary study," Engineering Journal, vol. 15, 2011.

[3] X. Zheng, X. Sun, K. Fu, and H. Wang, "Automatic annotation of satellite images via multifeature joint sparse coding with spatial relation constraint," IEEE Geoscience and Remote Sensing Letters, vol. 10, pp. 652-656, 2013.

[4] A. Karlsson, "Classification of high resolution satellite images," 2003. 
[5] P. Mather and B. Tso, Classification methods for remotely sensed data: CRC press, 2016.

[6] D. Lu and Q. Weng, "A survey of image classification methods and techniques for improving classification performance," International journal of Remote sensing, vol. 28, pp. 823-870, 2007.

[7] O. S. Soliman and A. S. Mahmoud, "A classification system for remote sensing satellite images using support vector machine with non-linear kernel functions," in Informatics and Systems (INFOS), 2012 8th International Conference on, 2012, pp. BIO-181-BIO-187.

[8] A. Elsharkawy, M. Elhabiby, and N. El-Sheimy, "New Combined Object-Based Technique for Efficient Urban Classsification Using WORLDVIEW-2 Data," ISPRSInternational Archives of the Photogrammetry, Remote Sensing and Spatial Information Sciences, vol. 1, pp. 191-195, 2012.

[9] L. Geosystems, "ERDAS field guide," Norcross,. Georgia: Leica Geosystems Geospatial Imaging, LLC, 2005.

[10] J. B. Campbell and R. H. Wynne, Introduction to remote sensing: Guilford Press, 2011.

[11] M. Nair and J. Bindhu, "Supervised Techniques and Approaches for Satellite Image Classification," International Journal of Computer Applications, vol. 134, 2016.

[12] M. Fauvel, "Spectral and spatial methods for the classification of urban remote sensing data," Institut National Polytechnique de Grenoble-INPG; Université d'Islande, 2007.

[13] D. T. Larose, Discovering knowledge in data: an introduction to data mining: John Wiley \& Sons, 2014.

[14] F. Al-Ahmadi and A. Hames, "Comparison of four classification methods to extract land use and land cover from raw satellite images for some remote arid areas, kingdom of Saudi Arabia," Earth, vol. 20, pp. 167-191, 2009.

[15] T. Hill, P. Lewicki, and P. Lewicki, Statistics: methods and applications: a comprehensive reference for science, industry, and data mining: StatSoft, Inc., 2006.

[16] S. M. Davis, D. A. Landgrebe, T. L. Phillips, P. H. Swain, R. M. Hoffer, J. C. Lindenlaub, et al., "Remote sensing: the quantitative approach," New York, McGrawHill International Book Co., 1978. 405 p., vol. 1, 1978.

[17] A. Asmala, "Analysis of maximum likelihood classification on multispectral data," Applied Mathematical Sciences, vol. 6, pp. 6425-6436, 2012.

[18] P. M. Mather and M. Koch, Computer processing of remotely-sensed images: an introduction: John Wiley \& Sons, 2011.

[19] J. Piper, "Variability and bias in experimentally measured classifier error rates," Pattern Recognition Letters, vol. 13, pp. 685-692, 1992.

[20] I. A. Huqqani and K. Khurshid, "Comparative Study of Supervised Classification of Urban Area Hyperspectral Satellite Imagery," Journal of Space Technology, vol. 4, pp. 7-14, 2014. 